\title{
Invisíveis a céu aberto: gestante em situação de rua
}

\author{
Invisible in the open: street pregnant woman \\ Invisible al aire libre: embarazada en la calle
}

Recebido: 19/03/2021 | Revisado: 26/03/2021 | Aceito: 29/03/2021 | Publicado: 08/04/2021

\author{
Débora Gabrielle da Rocha Lima \\ ORCID: https://orcid.org/0000-0002-3576-7047 \\ Universidade Estácio de Sá, Brasil \\ E-mail: gabi22lima@yahoo.com.br \\ Andressa Medeiros dos Santos \\ ORCID: https://orcid.org/0000-0001-7329-3092 \\ Universidade Estácio de Sá, Brasil \\ E-mail: ds_andressa@hotmail.com \\ Suzane Coelho Martins \\ ORCID: https://orcid.org/0000-0002-8980-2514 \\ Universidade Estácio de Sá, Brasil \\ E-mail: suzanecoelhoantonio@yahoo.com.br
}

\begin{abstract}
Resumo
No Brasil, a realidade vivida por pessoas em situação de rua é marcada pela grande desigualdade social. A incerteza gerada ao se viver nas ruas ocorre devido a exposições constantes a perigos. Trata-se de um grupo heterogêneo, que tem como vínculo a pobreza extrema, incluindo gestantes. O objetivo dessa pesquisa é caracterizar as vulnerabilidades vivenciadas pelas gestantes que vivem em situação de rua. Trata-se de uma pesquisa qualitativa descritiva de revisão integrativa, cujos aspectos facilitam na identificação dos resultados relevantes. A coleta das informações para a pesquisa bibliográfica foi feita através das bases de dados da Biblioteca Virtual em Saúde (BVS), acessada por meio do Banco de Dados em Enfermagem (BDENF); Biblioteca Científica Eletrônica Virtual (SCIELO), Literatura Latino Americana e do Caribe em Ciências da Saúde (LILACS) e Google scholar. A partir dessa pesquisa espera-se contribuir e ampliar o debate sobre as dificuldades de gestantes em situação de rua, os obstáculos enfrentados pelas equipes multiprofissionais do consultório de rua. A busca localizou 16 artigos dentro do tema, publicados entre 2015 e 2019. Estes foram divididos em três categorias: gestantes usuárias de drogas, seu cotidiano e a importância do prénatal, e o acesso a saúde desse grupo. Concluiu-se que o pouco conhecimento dos profissionais de saúde, sobre as dificuldades enfrentadas por essas gestantes, interfere no segmento e adesão ao pré-natal. A construção de um relacionamento com essas gestantes é indispensável, pois ajuda na melhoria do serviço que atende esse público.
\end{abstract}

Palavras-chave: População de rua; Gestante; Vulnerabilidade social; Consultório de rua.

\begin{abstract}
In Brazil, the reality experienced by homeless people is marked by great social inequality. The uncertainty generated by living on the streets is due to constant exposure to dangers. It is a heterogeneous group, linked to extreme poverty, including pregnant women. The objective of this research is to characterize the vulnerabilities experienced by pregnant women living on the streets. It is a qualitative descriptive research of an integrative review; whose aspects facilitate the identification of relevant results. The collection of information for bibliographic research was done through the databases of the Virtual Health Library (VHL), accessed through the Nursing Database (BDENF); Virtual Electronic Scientific Library (SCIELO), Latin American and Caribbean Literature in Health Sciences (LILACS) and Google scholar. From this research it is expected to contribute and expand the debate on the difficulties of pregnant women on the street, the obstacles faced by the multiprofessional teams of the street office. The search found 16 articles on the topic, published between 2015 and 2019. These were divided into three categories: pregnant women who use drugs, their daily lives and the importance of prenatal care, and access to health for this group. It was concluded that the little knowledge of health professionals, about the difficulties faced by these pregnant women, interferes in the segment and adherence to prenatal care. Building a relationship with these pregnant women is essential, as it helps to improve the service that serves this audience.
\end{abstract}

Keywords: Street population; Pregnant; Social vulnerability; Street office.

\section{Resumen}

En Brasil, la realidad que viven las personas sin hogar está marcada por una gran desigualdad social. La incertidumbre que genera vivir en la calle se debe a la exposición constante a los peligros. Es un grupo heterogéneo, vinculado a la pobreza extrema, incluidas las mujeres embarazadas. El objetivo de esta investigación es caracterizar las vulnerabilidades que experimentan las mujeres embarazadas que viven en la calle. Se trata de una investigación 
descriptiva cualitativa de revisión integradora, cuyos aspectos facilitan la identificación de resultados relevantes. La recolección de información para la investigación bibliográfica se realizó a través de las bases de datos de la Biblioteca Virtual en Salud (BVS), a las que se accede a través de la Base de Datos de Enfermería (BDENF); Biblioteca Científica Electrónica Virtual (SCIELO), Literatura Latinoamericana y del Caribe en Ciencias de la Salud (LILACS) y Google Scholar. A partir de esta investigación se espera contribuir y ampliar el debate sobre las dificultades de las mujeres embarazadas en la calle, los obstáculos que enfrentan los equipos multiprofesionales de la oficina de calle. La búsqueda encontró 16 artículos sobre el tema, publicados entre 2015 y 2019. Estos se dividieron en tres categorías: mujeres embarazadas que consumen drogas, su vida diaria y la importancia de la atención prenatal y el acceso a la salud para este grupo. Se concluyó que el poco conocimiento de los profesionales de la salud, sobre las dificultades que enfrentan estas gestantes, interfiere en el segmento y adherencia a la atención prenatal. Construir una relación con estas embarazadas es fundamental, ya que ayuda a mejorar el servicio que atiende a esta audiencia.

Palabras clave: Población callejera; Embarazada; Vulnerabilidad social; Oficina en la calle.

\section{Introdução}

No Brasil, a realidade vivida por pessoas em situação de rua é marcada pela grande desigualdade social; sendo essa uma situação não apenas vista atualmente. Desde o princípio da era industrial, o agrupamento de migrantes para as grandes cidades estabelece cenários de moradia dessas pessoas nas ruas, uma vez que, não havia trabalho e residência para todos. Contudo, a nova população em situação de rua surgiu do segmento similarmente de migração e partida rural, que segue o crescimento das cidades em razão da falta de oportunidade em cidades menores (Vale e Vecchia, 2019).

É neste cenário que surge a População em Situação de Rua (PSR), comunidade historicamente desprezada que vive o estereótipo de repúdio, sem documentos, sujos, desprezados e sem respeitabilidade. Encontram-se na margem dos processos de integração e suportam níveis variados de vulnerabilidade e hostilidade, pouco relatada, porém, muito vista nas ruas, faróis, centros de cidades, áreas faveladas e casa de apoio. É qualificada como população flutuante (Lira, et al., 2019).

A situação incerta e doentia das ruas dá-se em exposições e perigos acumulados, sobre as quais são indispensáveis procedimentos e formas de intervenção que atendam às particularidades de cada ocorrência. Diante disso, os princípios de universalidade, integralidade e equidade do Sistema Único de Saúde (SUS) são um estímulo para efetivação de políticas públicas de saúde para a PRS (Medeiros e Cavalcante, 2018).

O sistema único de saúde (SUS) foi fundamental na esfera das políticas públicas no Brasil, considerando a participação popular essencial, reiterando e destacando a importância do controle social e práticas de saúde (Cardoso, et al., 2018).

Entendendo que todas as pessoas têm direito a ações e serviços de saúde, intensificando o entendimento da saúde como direito de todos e dever do estado (Lira, et al., 2019).

A população de rua se caracteriza como um grupo heterogêneo, que tem como vínculo a pobreza extrema, relacionamentos familiares e comunitários bastante fragilizados ou rompidos e moradia não habitual, de maneira que, buscam espaços públicos ou áreas degradadas para esse fim. Essa circunstância pode ser vista como temporária ou definitiva (Paula, et al., 2018).

Segundo Rosa e Brêtas (2015) "a condição de morador de rua expõe o sujeito ao enfrentamento de carências de toda a ordem, além de exigir que ao mesmo tempo ele se adapte a outras referências de vida social bastante distinta daquelas anteriormente vividas',

um estudo realizado em 71 cidades brasileiras traçou o perfil da população em situação de rua, de acordo com alguns aspectos como: quanto à idade, mostrou que 30\% da população que vive nas ruas têm entre 26 e 35 anos, $39,1 \%$ se declaram parda, a maioria dessas não concluiu o ensino fundamental, e 63,5 ressalta que são alfabetizadas (Santana, et al., 2019). 
As mulheres são minoria entre a PRS, onde são as principais vítimas de violência e práticas constante de sexo, por vezes sem método de contracepção, em troca de drogas e alimento (Biscotto, et al., 2015).

gestantes que vivem nas ruas estão sujeitas a vários tipos de riscos, podendo-se citar acompanhamento pré-natal inadequado, presença de Infecções Sexualmente Transmissíveis (IST), por vezes associadas ao abandono de tratamento, o uso de álcool e outras drogas, idade gestacional incerta, alimentação escassa, higiene inadequada, entre outros (Almeida \& Quadros, 2016).

A chegada de uma criança se exige um lar, uma família, recepção estruturada, mais a realidade e diferente para essas gestantes que vivem em situação de rua, pois elas vivenciam situações desfavoráveis, muitas são usuárias de drogas, com situação financeira frágil, longe de um cenário ideal para se construir uma família (Costa, et al., 2015).

Conforme as políticas existentes no âmbito do Sistema Único de Saúde (SUS), gestores e profissionais de saúde necessitam estarem alerta às especificidades apresentadas por essas gestantes em situação de rua, com intuito de acessibilidade de ações e serviços de saúde. A atenção básica tem como sua principal estratégia os consultórios na rua que são formados por equipes multiprofissionais que desenvolvem atividades completas de saúde, com a finalidade o cuidado da população em situação de rua, e inserção à saúde (Silva, 2019).

As equipes multidisciplinares do programa consultório de rua atuam na atenção básica de saúde, oferecendo atendimento clínico e psicossocial, oferecendo cuidados em saúde para essas gestantes que vivem em vulnerabilidade (Silva, 2019).

Estudos apontam que quando há assistência de qualidade durante o pré-natal, se tem resultados melhores durante o parto, $\log$ as ações educativas realizadas durante o ciclo gravídico são valorosas, pois a gestante possui menos riscos de complicações (Araújo, et al., 2017).

A realização do pré-natal é essencial e pode ser realizada pelo consultório de rua com apoio de hospitais e maternidades, em casos de alto risco. A percepção da gestante, com intuito de aproximação e vínculo é um ponto crítico há muita resistência, pois é visto como uma exposição da sua condição de vida (Araújo, et al., 2017).

Compreender a vivência das gestantes vulneráveis propicia elementos para a prática de um cuidado humanizado e compreensivo, é necessário analisar várias questões, pois estas mulheres podem manifestar diversas queixas no decorrer da assistência a sua gestação (Lima, et al., 2015).

\section{Metodologia}

Trata- se de uma pesquisa qualitativa descritiva de revisão integrativa. Neste estudo são percorridas as seguintes etapas (Souza, et al., 2010): $1^{\mathrm{a}}$ fase: Elaboração da pergunta norteadora, $2^{\mathrm{a}}$ fase: busca ou amostragem na literatura, $3^{\mathrm{a}}$ fase: coleta de dados, $4^{\mathrm{a}}$ fase: análise crítica dos estudos incluídos, $5^{\mathrm{a}}$ fase: discussão dos resultados, $6^{\mathrm{a}}$ fase: apresentação da revista integrativa.

A Revisão Integrativa é um método de pesquisa apontado como ferramenta de grande relevância no campo da saúde, por proporcionar a busca, avaliação crítica e a síntese de evidências sobre um tema investigado. Esses aspectos facilitam a identificação dos resultados relevantes, de lacunas que direcionam para o desenvolvimento de futuras pesquisas e auxiliam o profissional a escolher condutas e a tomar decisões, proporcionando um saber crítico (Souza, et al., 2010).

Segundo Bardin (2011) as categorias devem possuir certas qualidades como: exclusão mútua, cada elemento só pode existir em uma categoria; homogeneidade, para definir uma categoria é preciso haver só uma dimensão na análise, se existe diferentes níveis de análise, elas devem ser separadas em diferentes categorias; pertinência as categorias devem dizer respeito às intenções do investigador, aos objetivos da pesquisa ás questões norteadoras; objetividade e fidelidade se as categorias 
forem bem definidas; produtividade as categorias serão produtivas se os resultados forem férteis em interferência, em hipóteses novas em dados exatos.

\section{Questão Norteadora}

A questão norteadora foi construída de forma objetiva, vinculada a um raciocínio teórico.

Desta forma explorara publicações cientificas sobre as dificuldades e vulnerabilidades vividas pelas gestantes que vivem em situação de rua. Ela encontra-se no capitulo um do presente trabalho.

Segundo Araújo (2020) a estratégia de PICo considera outros blocos temáticos para a construção de estratégia de busca com objetivo de recuperação da informação em saúde.

Ela considera a população, ou paciente ou o problema abordado (population,patient,problem), o fenômeno de interesse (Interest) e o contexto ( Context).

Quadro 1: Componentes da pergunta da pesquisa anagrama de PICo.

\begin{tabular}{|l|l|l|}
\hline Acrônimo & Descrição & \multicolumn{1}{|c|}{ Componentes da questão } \\
\hline P & População & Gestante \\
\hline I & Interesse & Os desafios e as vulnerabilidades. \\
\hline Co & Contexto & Situação de rua \\
\hline
\end{tabular}

Fonte: Saúde e medicina (2014)

\section{Buscas na literatura - amostragem}

A coleta das informações para a pesquisa bibliográfica ocorreu por meio da exploração da base de dados da Biblioteca Virtual em Saúde (BVS), acessada por meio do Banco de Dados em Enfermagem (BDENF), Biblioteca Científica Eletrônica Virtual (SCIELO), Literatura Latino Americana e do Caribe em Ciências da Saúde (LILACS) e Google scholar.

Empregou-se o operador booleano "AND" durante a pesquisa.

Foram pesquisados artigos publicados em língua portuguesa, nos últimos cinco anos, texto completo, disponível online, com acesso livre. Foram excluídos da amostra os artigos publicados em línguas estrangeiras, artigos repetidos e artigos que não abordassem a temática do estudo.

A busca na base de dados foi orientada pelos descritores: População em Situação de Rua, Gestante, Vulnerabilidade Social, Consultório de Rua, foi realizada em todos os índices, buscando captar o maior número de artigos publicados no período proposto que abordasse a temática em discussão.

Instrumentos de coleta de dados

Para essa etapa do projeto foi elaborado um instrumento para a consolidação dos dados no programa Microsoft Word2007 em formato de planilha para organizar adequadamente a extração das informações dos estudos selecionados no intuito de facilitar a análise das amostras extraídas.

O instrumento apresenta as seguintes informações: título do artigo, ano de publicação do artigo, base de dados, objetivos propostos e Qualis da revista.

Essa etapa faz-se necessária, pois a mesma irá determinar a confiança dos resultados e fortalecer as conclusões sobre o estado atual do tema investigado (Souza, et al., 2010).

Para uma análise crítica e reflexiva dos estudos incluídos na revisão, foi realizada uma leitura minuciosa e criteriosa destacando os que atingiram os critérios de inclusão e que contemplaram o objetivo proposto, viabilizando o resultado da pesquisa de forma clara e objetiva. 
Segundo Bardin (2011) indica que a utilização da análise de conteúdo prevê três fases fundamentais: pré-análise exploração do material e tratamento dos resultados.

A primeira fase é a pré-análise onde é identificada como a fase de organização. Nela se estabelece um esquema de trabalho preciso e bem definido.

A segunda fase é a exploração do material consiste em definir as categorias e a identificação das unidades de registro.

A terceira fase consiste no tratamento dos resultados, interpretação.

Figura 1: Fluxograma de etapas de avaliação dos artigos.

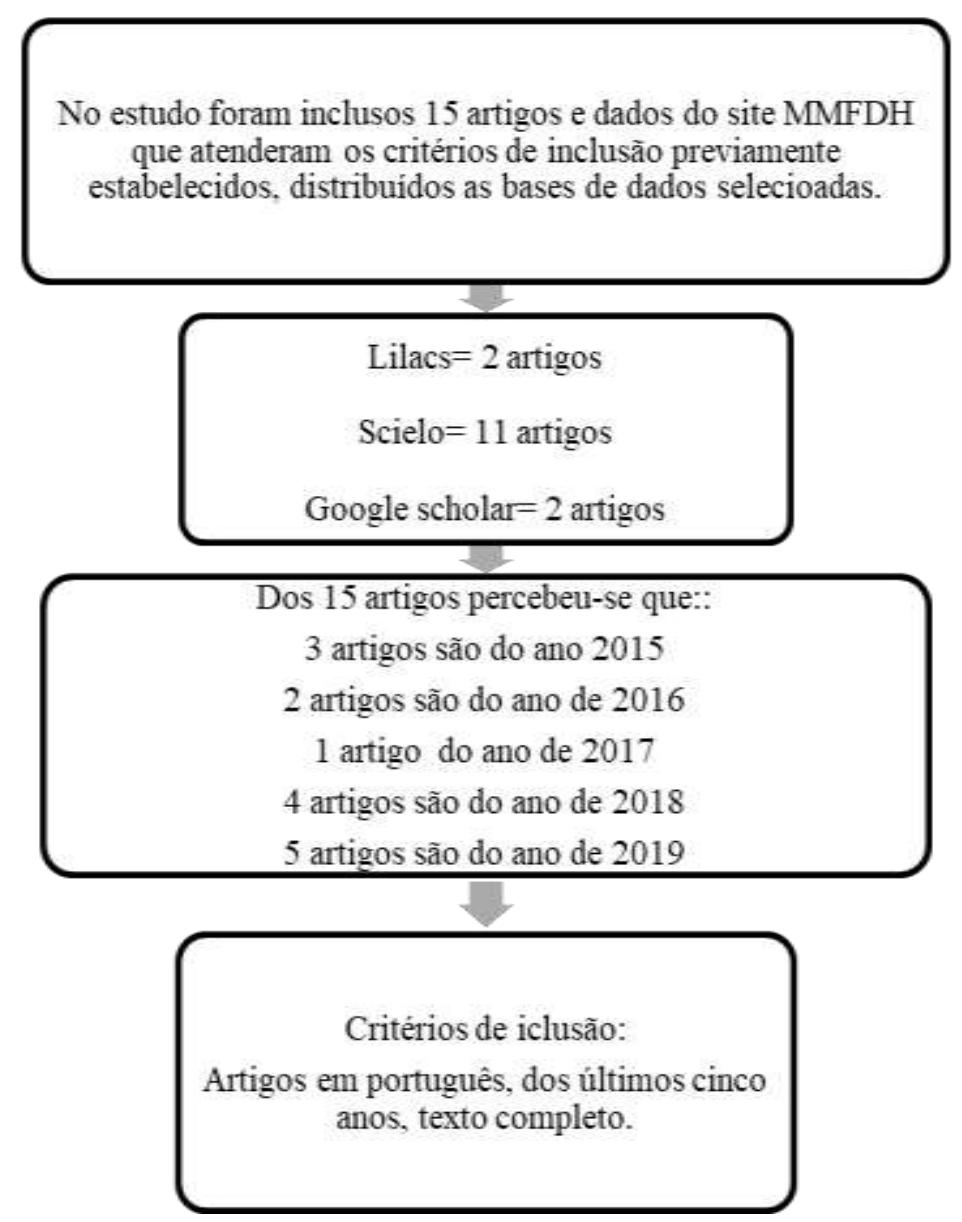

. Fonte: Elaboração própria (2020).

Para a análise dos dados foi utilizado à análise temática defendida por Bardin (2011), caracterizando em formar uma unidade temática e categorias no agrupamento dos assuntos trazido nos artigos selecionados.

\section{Resultados e Discussão}

Para fazer jus a análise de Bardin, desenhamos a unidade temática, que na leitura e releitura dos artigos foi descrita como: A vida nas ruas e seus desafios, e a escassez de políticas de saúde voltadas a essa população, as categorias, descritas abaixo, que respondem a pesquisa. 
Quanto aos assuntos abordados pelos artigos foi observado que dois artigos falam sobre gestantes em situação de rua usuária de drogas; seis artigos falam sobre a mulher em situação de rua, ciclo gravídico em situação de rua e importância do pré-natal para gestantes que vivem em situação de rua; sete artigos falam sobre o acesso a saúde da população em situação de rua e o consultório de rua. No gráfico abaixo consta a distribuição anual dos artigos analisados.

Gráfico 1: problemática da PSR.

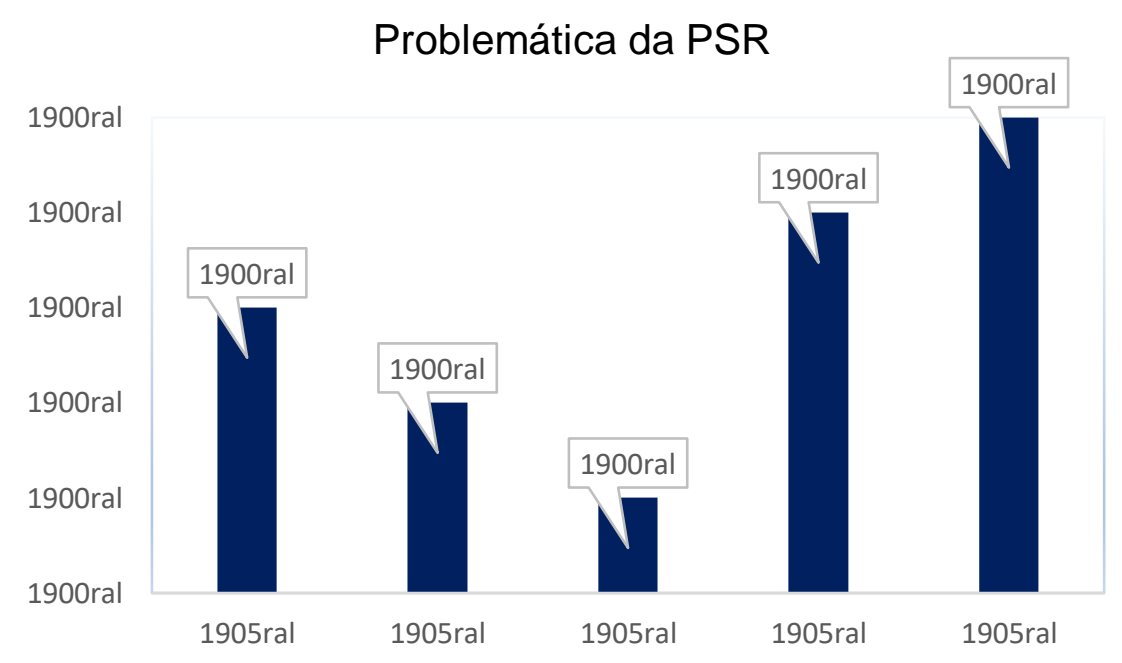

Fonte: Elaboração própria (2020).

Os resultados foram separados em três categorias, a primeira categoria trata das gestantes usuárias de drogas que vivem em situação de rua. A segunda categoria apresenta os artigos que tratam do enfrentamento da maternidade nas ruas, mulher em situação de rua no ciclo gravídico, seu cotidiano e a importância do pré-natal. A terceira e última categoria traz a questão do consultório de rua, o acesso a saúde da população que vive em situação de rua.

Categoria 1: Gestantes em situação de rua usuária de drogas

Nesta categoria foram encontrados dois artigos conforme Tabela 1. Um dos artigos trata das experiências vividas pelas gestantes moradoras de rua e todas as dificuldades pelas quais elas passam nessa situação, o outro traz o papel dos profissionais de enfermagem no amparo a ser dado as gestantes, usuárias de drogas durante a gestação. Ambos possuem classificação Qualis B e foram publicados em revistas nacionais. 
Tabela 1: Artigos sobre gestantes em situação de rua usuária de drogas.

\begin{tabular}{l|l|l|l|l|l}
\hline Título do artigo & Objetivo \\
\hline A pedra que pariu & $\begin{array}{l}\text { Apresentar experiência } \\
\text { vivida por mulheres em }\end{array}$ & $\begin{array}{l}\text { Pesquisas e } \\
\text { Práticas } \\
\text { situação de rua que utilizam } \\
\text { Psicossociais } \\
\text { drogas }\end{array}$ & $\begin{array}{l}\text { B2 } \\
(2016) .\end{array}$ & SCIELO \\
\hline O papel do enfermeiro & $\begin{array}{l}\text { Analisar as ações de } \\
\text { enfermagem prestadas no }\end{array}$ & $\begin{array}{l}\text { Espaço Saúde } \\
(2015) .\end{array}$ & B5 & LILACS \\
nurante a consulta de pré- \\
drogas
\end{tabular}

Fonte: Elaboração própria (2020).

No Brasil, o consumo de drogas lícitas e ilícitas usadas pela população feminina tem aumentado significativamente ao longo dos anos. Presume-se que aproximadamente $20 \%$ das mulheres usam drogas durante a gravidez, o uso constante tem aumentado nos últimos anos (Lima et al., 2015).

Embora menores quantitativamente, essas gestantes possuem um histórico frequente de violência, e a sua permanência na rua é atravessada por fragilidades e pela prática constante de sexo, muitas vezes sem método contraceptivo em troca de drogas (Almeida \& Quadros, 2016).

Algumas drogas passam livre através da placenta, como exemplo, o consumo de álcool durante a gestação (Almeida e QuadroS, 2016).

A utilização de álcool, fumo e outras drogas durante a gestação têm levado ao crescimento das alterações fetais e neonatais. Para reduzir os efeitos tóxicos das drogas na gestação é primordial a participação de um bom programa de assistência à gestante, associando as consultas de pré-natal, ao CAPS/AD (Lima et al., 2015).

Promover a saúde das gestantes é proporcionar o ciclo gravídico-puerperal de modo que ele ocorra de maneira mais tranquila e segura (Lima et al., 2015).

É importante reconhecer as dificuldades visíveis no cotidiano das gestantes em situação de rua, para iniciar ou dar sequência a assistência pré-natal, ressaltando a relevância desse acesso e assistência diante do comportamento de uso de drogas ao longo desse período (Almeida \& Quadros, 2016).

A construção de um processo gradual, lento e cuidadoso de aproximação, vínculo e reconhecimento de afetos, é fundamental para a relação com a gestante que vive em situação de rua (Almeida \& Quadros, 2016).

Categoria 2: Enfrentando a maternidade nas ruas, mulher em situação de rua no ciclo gravídico, seu cotidiano e a importância do pré- natal.

$\mathrm{Na}$ Tabela 2 constam os artigos que tratam da mulher em situação de rua, durante o ciclo gravídico e a importância do pré-natal. Todos os seis artigos possuem classificação Qualis B e foram publicados em revistas nacionais. 
Tabela 2: Artigos sobre gestantes em situação de rua e a importância do pré-natal.

\begin{tabular}{|c|c|c|c|c|}
\hline Trítulo do artigo & Objetivo & Revista/ ano & Qualis & Base de Dados \\
\hline $\begin{array}{l}\text { A violência na vida } \\
\text { das mulheres em } \\
\text { situação de rua na } \\
\text { cidade de São Paulo }\end{array}$ & $\begin{array}{l}\text { Trazer reflexões, situações de violência } \\
\text { na vida de mulheres em situação de rua. }\end{array}$ & $\begin{array}{l}\text { Interface } \\
\text { Comunicação, } \\
\text { Saúde, Educação } \\
\text { (2015). }\end{array}$ & B1 & SCIELO \\
\hline $\begin{array}{l}\text { Compreensão da } \\
\text { vivência de mulheres } \\
\text { em situação de rua. }\end{array}$ & $\begin{array}{l}\text { Compreender a experiência de vida de } \\
\text { mulheres em situação de rua. }\end{array}$ & $\begin{array}{l}\text { Revista da } \\
\text { Escola de } \\
\text { Enfermagem da } \\
\text { USP (2016). }\end{array}$ & B2 & SCIELO \\
\hline $\begin{array}{l}\text { O contexto da } \\
\text { gestante na situação } \\
\text { de rua } \quad \text { e } \\
\text { vulnerabilidade: seu } \\
\text { Olhar sobre o pré- } \\
\text { natal }\end{array}$ & $\begin{array}{l}\text { Investigar como ocorrem os cuidados } \\
\text { de Enfermagem diante da condição de } \\
\text { risco relacionada ao Período } \\
\text { gestacional no contexto de situação de } \\
\text { vulnerabilidade social. }\end{array}$ & $\begin{array}{l}\text { Revista de } \\
\text { Enfermagem } \\
\text { UFPE (2017). }\end{array}$ & B2 & $\begin{array}{l}\text { LILACS } \\
\text { BDENF }\end{array}$ \\
\hline $\begin{array}{l}\text { Maternidade } \\
\text { adolescente } \\
\text { contexto das ruas }\end{array}$ & $\begin{array}{l}\text { Discutir práticas em curso destinadas às } \\
\text { adolescentes grávidas e/ou mães em } \\
\text { situação de rua. Aspectos positivos da } \\
\text { maternidade, a partir dos discursos das } \\
\text { jovens mães, ainda que seja } \\
\text { reconhecida a complexidade do } \\
\text { cotidiano de vida dessas adolescentes, a } \\
\text { escassez de serviços específicos } \\
\text { voltados para seu atendimento }\end{array}$ & $\begin{array}{l}\text { Desidades } \\
(2018) \text {. }\end{array}$ & B4 & SCIELO \\
\hline $\begin{array}{l}\text { Gestantes } \\
\text { situação de rua no } \\
\text { município de Santos, } \\
\text { SP: reflexões e } \\
\text { desafios para as } \\
\text { políticas públicas }\end{array}$ & $\begin{array}{l}\text { Investigar o cotidiano de gestantes em } \\
\text { situação de rua e sua relação com as } \\
\text { políticas públicas na cidade de Santos, } \\
\text { litoral do Estado de São Paulo. }\end{array}$ & $\begin{array}{l}\text { Saúde } \\
\text { Sociedade } \\
(2019)\end{array}$ & B1 & SCIELO \\
\hline $\begin{array}{l}\text { Assistência de } \\
\text { enfermagem a } \\
\text { mulher em situação } \\
\text { de rua no ciclo- } \\
\text { gravídico puerperal }\end{array}$ & $\begin{array}{l}\text { Identificar à assistência de enfermagem } \\
\text { a mulher em situação de rua }\end{array}$ & $\begin{array}{l}\text { Ciências } \\
\text { Biológicas e de } \\
\text { Saúde UNIT } \\
\text { (2019). }\end{array}$ & B5 & $\begin{array}{l}\text { GOOGLE } \\
\text { SCHOLAR }\end{array}$ \\
\hline
\end{tabular}

Fonte: Elaboração própria (2020).

A gestação é um momento único na vida de uma mulher, durante esse período, acontecem várias mudanças diariamente, podendo ser biológicas, psicológicas e sociais (Costa et al., 2015). 
Quando a mulher engravida e não possui um lar, a situação se torna desfavorável, já que é um momento de fragilidade, ela precisa de um abrigo adequado (Santana et al.,2019).

Nas ruas, o acesso aos serviços públicos e as viabilidades de apoio e melhoria de vida é limitado o que torna a realidade mais desafiadora (Rizzini, 2018).

A realidade de estar nas ruas acaba expondo a gestante a múltiplos riscos, além de dificultar seu vínculo a um serviço de saúde (Costa et al., 2015).

A vida nas ruas acaba ocasionando envolvimento com prostituição, uso abusivo de álcool, drogas e a vários tipos de violência causando riscos para a saúde materno-infantil (Santana et al.,2019).

As gestantes possuem vulnerabilidades peculiares da gravidez quando vivenciada nas ruas torna-se um elemento de risco para mãe e feto (Santana et al.,2019).

O consumo de drogas faz parte do cotidiano das gestantes que vivem em situação de rua tornando-se um vício, pois o efeito de algumas substâncias diminui alguns problemas como frio, fome, dor e sono (Costa, et al., 2015).

O monitoramento da gestante usuária de drogas deve ser por meio de estratégias voltadas para a identificação precoce nos serviços de saúde, por possuírem risco de complicações maternas e fetais (Araújo, et al., 2017).

Com o propósito de reduzir as taxas de mortalidade materna- infantil foi elaborado o programa de humanização no pré-natal e nascimento, com a finalidade de proporcionar o desenvolvimento da gestação garantindo o parto de um recémnascido saudável sem impacto para a saúde materna (Santana, et al.,2019).

O número de consultas pré-natal por maior que seja não assegura que a assistência seja adequada, o que avalia é a qualidade das consultas como a escuta das gestantes esclarecimentos de dúvidas, atividades assistenciais e educativas, informações necessárias sobre a gravidez, procedimentos indispensáveis como exames básicos laboratoriais, imunização e teste HIV (Araújo, et al., 2017).

Segundo Rosa e Brêtas (2015) enquanto profissionais que assistimos esta população, devemos estar atentos ao impacto que os modos de vida na rua têm sobre o processo saúde-doença-cuidado para estas pessoas.

$\mathrm{Na}$ consulta deve-se ter acolhimento da gestante, buscado manter um diálogo que permite a livre manifestação da fala e sentimento. Com isso é possível compreender que a comunicação é um suporte para a relação entre o profissional e a gestante (Santana, et al.,2019).

O cuidado humanizado e compreensivo dos profissionais de saúde favorece na implementação de estratégias que ajudam a vencer o desalento de mulheres que se sentem esquecidas devido a sua vulnerabilidade e sua situação de pobreza (Araújo, et al., 2017).

Segundo Biscotto, et al., (2015)

A perspectiva da fenomenologia social trouxe como contribuições a possibilidade de acessar a intersubjetividade que permeia o cotidiano das mulheres em situação de rua, revelando questões complexas e conflituosas vivenciadas nesse contexto social. Tal complexidade requer o direcionamento de olhares e práticas que auxiliem no atendimento das necessidades biopsicossociais deste público.

Estar em situação de rua significa viver em condição de extrema pobreza e vivenciar constantemente a violação de direitos, ocasionando a exclusão de direitos básicos como moradia, alimentação, educação, higiene entre outros, situação comum de quem utiliza de locais públicos como espaço de sobrevivência (Santana, et al.,2019).

Categoria 3: Consultório de rua, o acesso a saúde da população que vive em situação de rua.

$\mathrm{Na}$ Tabela 3 constam os artigos que tratam sobre o consultório de rua, seu atendimento. Os artigos possuem Qualis A e os demais Qualis B, todos publicados em revistas nacionais. 
Tabela 3: Artigos sobre consultório de rua, acesso a saúde e políticas públicas, voltadas à população em situação de rua.

\begin{tabular}{|c|c|c|c|c|}
\hline Título do artigo & Objetivo & Revista/ ano & Qualis & Base de Dados \\
\hline $\begin{array}{l}\text { Desafios e potencialidades do } \\
\text { trabalho de enfermagem em } \\
\text { consultório de rua }\end{array}$ & $\begin{array}{l}\text { Analisar elementos do processo } \\
\text { de trabalho de enfermagem no } \\
\text { Consultório de rua desafios e } \\
\text { potencialidade nos cuidados a } \\
\text { pessoa em situação de rua }\end{array}$ & $\begin{array}{l}\text { Revista Latino- } \\
\text { Americana de } \\
\text { Enfermagem } \\
\text { (2018). }\end{array}$ & B2 & SCIELO \\
\hline $\begin{array}{l}\text { A implantação } \\
\text { Consultório na Rua na } \\
\text { Perspectiva Do cuidado em } \\
\text { saúde }\end{array}$ & $\begin{array}{l}\text { Relatar a experiência sobre a } \\
\text { implantação de Consultório na } \\
\text { Rua }\end{array}$ & $\begin{array}{l}\text { Revista } \\
\text { Brasileira de } \\
\text { Enfermagem } \\
\text { (2018). }\end{array}$ & $\mathrm{A} 2$ & SCIELO \\
\hline $\begin{array}{l}\text { A implementação do } \\
\text { programa de saúde } \\
\text { específico para a população } \\
\text { em situação de rua - } \\
\text { Consultório na rua: barreiras } \\
\text { e facilitadores }\end{array}$ & $\begin{array}{l}\text { Investigar as razões da baixa } \\
\text { adesão à implementação do } \\
\text { programa de saúde específico } \\
\text { para a população em situação de } \\
\text { rua }\end{array}$ & $\begin{array}{l}\text { Saúde } \\
\text { Sociedade } \\
\text { (2018). }\end{array}$ & B1 & SCIELO \\
\hline $\begin{array}{l}\text { "UPA é nós aqui mesmo": as } \\
\text { redes de apoio social no } \\
\text { cuidado à saúde da } \\
\text { população em situação de } \\
\text { rua em um município de } \\
\text { pequeno porte }\end{array}$ & $\begin{array}{l}\text { Identificar e analisar o itinerário } \\
\text { terapêutico da população em } \\
\text { situação de rua. }\end{array}$ & $\begin{array}{l}\text { Saúde e } \\
\text { Sociedade } \\
\text { (2019). }\end{array}$ & B1 & SCIELO \\
\hline $\begin{array}{l}\text { Consultório na rua: } \\
\text { Visibilidades, invisibilidade } \\
\text { e hipervisibilidade. }\end{array}$ & $\begin{array}{l}\text { Refletir sobre o acolhimento e a } \\
\text { produção de cuidados } \\
\text { destinados a população em } \\
\text { situação de rua. }\end{array}$ & $\begin{array}{l}\text { Cadernos de } \\
\text { Saúde Pública } \\
(2015) \text {. }\end{array}$ & $\mathrm{A} 2$ & SCIELO \\
\hline $\begin{array}{l}\text { O acesso da população em } \\
\text { situação de rua é um direito } \\
\text { negado? }\end{array}$ & $\begin{array}{l}\text { Analisar o acesso da população } \\
\text { em situação de rua (PSR) aos } \\
\text { serviços de saúde. }\end{array}$ & REME (2019). & B1 & SCIELO \\
\hline $\begin{array}{l}\text { A cobertura do programa } \\
\text { consultório na rua e o } \\
\text { impacto no acesso da } \\
\text { população em situação de } \\
\text { rua aos serviços de saúde }\end{array}$ & $\begin{array}{l}\text { Estudo sobre programa } \\
\text { consultório na rua e como sua } \\
\text { implantação influenciou a } \\
\text { população no acesso aos } \\
\text { serviços de saúde. }\end{array}$ & LUME (2019). & B4 & $\begin{array}{l}\text { GOOGLE } \\
\text { SCHOLAR }\end{array}$ \\
\hline
\end{tabular}

Fonte: Elaboração própria (2020).

A População em Situação de Rua (PSR) está, historicamente, incluída nas diferentes sociedades como um grupo que possui muitas vulnerabilidades (Paula, et al., 2018). 
As condições incertas e insalubres das ruas acontecem em exposições e riscos aglomerados, sobre os quais são necessárias ações e formas de tratamento que atendam às particularidades de cada situação (Medeiros \& Cavalcante, 2018).

São diversas exclusões vivenciadas diariamente por esse público, entre elas encontra-se a dificuldade de acesso aos serviços de saúde (Vale \& Vecchia, 2019).

A saúde é um direito de todos os brasileiros garantido pela Constituição Federal de 1988. Todo cidadão tem acesso ao Sistema Único de Saúde (SUS), realizado através de ações de prevenção, promoção e assistência à saúde para garantir esse direito, o SUS busca ampliar a facilidade de ingresso para todas as pessoas na rede de saúde. Contudo, alguns cidadãos estão em situação de vulnerabilidade e sofrem com muitas dificuldades e obstáculos para conseguir acesso aos serviços de saúde, sobretudo aqueles indivíduos que vivem em situação de rua (Lira, et al., 2019).

Segundo Silva (2019) o programa Consultório na Rua é uma estratégia que compõe a Política Nacional de Atenção Básica, que foi criada em 2011 com o objetivo de facilitar o acesso e o atendimento de pessoas em situação de rua.

O Consultório na rua é um equipamento itinerante de saúde que abrange a Rede de Atenção Básica e desenvolve ações de atenção psicossocial, devendo seguir os fundamentos e as diretrizes estabelecidas pela Política Nacional de Atenção Básica. Portanto, saúde é com os Centros de Atenção Psicossocial, priorizando que a assistência e o cuidado sejam realizados no próprio território de rua (Hallais \& Barros, 2015).

Compete às equipes de Consultório na rua proporcionar atenção à saúde em um contexto distinto e dinâmico, que desafia diariamente os profissionais de saúde que auxiliam os usuários em casos que fogem do convencional (Cardoso, et al., 2018).

As equipes dos Consultórios na rua são multiprofissionais e lidam com os diferentes problemas e carências de saúde apresentados pela população em situação de rua, evolvendo a busca ativa e o cuidado aos usuários de álcool, crack e outras drogas (Medeiros \& Cavalcante, 2018).

A população em situação de rua tem dificuldade de acesso aos serviços de saúde, referindo-se a burocratização no atendimento, em função da falta do cartão SUS e de endereço fixo da PSR, Outra dificuldade é o pouco conhecimento que a população em situação de rua tem sobre a organização dos serviços de saúde (Lira, et al., 2019).

O cuidado com a população em situação de rua demanda ampliação do olhar acerca do processo saúde-doençacuidado, assim como a utilização de diversos recursos que valorizem as pessoas e suas necessidades, tendo em consideração o território e suas singularidades (Cardoso, et al., 2018).

\section{Conclusão}

O desequilíbrio social e o pouco conhecimento dos profissionais de saúde, sobre as dificuldades enfrentadas pela gestante que vive em situação de rua, interferem no segmento e adesão ao pré-natal. A construção de um relacionamento com as gestantes é indispensável, pois ajuda na melhoria do serviço que atende esse público entendendo suas necessidades.

No estudo foram destacadas as dificuldades e as vulnerabilidades vivenciadas por gestantes que vivem em situação de rua. Essas mulheres fazem uso abusivo de drogas, são vítimas de violência se tornando vulnerável aos riscos.

O período gestacional é o momento em que a mulher se torna mais vulnerável psicologicamente e fisicamente, sendo assim as ruas não é um local adequado para se viver a maternidade, essa gestante precisa de cuidados, alimentação adequada, um acompanhamento fixo durante a gestação, o que nas ruas elas não têm.

Cabe ressaltar que as políticas públicas que cuidam dessa população são pouco empregadas, não há um planejamento que acolha as necessidades dessa população. 
Foi obtido um entendimento acerca da realidade das gestantes, seu cotidiano e o que sentem ao procurar um serviço de saúde.

Com a pesquisa foi possível obter respostas sobre as dificuldades e vulnerabilidades vivenciadas pelas gestantes em situação de rua, como é realizado o acompanhamento gestacional e as equipes que cuidam dessa população como fazem esse atendimento.

\section{Referências}

Almeida, D. J. R. \& Quadros, L. C. T. ( 2016). A pedra que pariu: Narrativas e práticas de aproximação de gestantes em situação de rua e usuárias de crack na cidade do Rio de Janeiro. Pesquisa prática psicossociais, São João Del-Rei, 11, (1), 225-237.

Araújo, A. S., Santos, A. A. P., Lúcio, I. M. L., Tavares, C. M. \&Fidélis, E. P. B.(2017). O contexto da gestante na situação de rua e vulnerabilidade: seu olhar sobre o pré-natal. Revista de enfermagem. UFPE . 11(supl.10), 4103-4110.

Biscotto, P. R., Jesus, M. C. P., Silva, M. H., Oliveira, D. M. \& Merighi, M. A. B.(2016). Compreensão da vivência de mulheres em situação de rua. Revista da escola de enfermagem. USP. 50(5). 749-755.

Cardoso, A. C., Santos, D. S., Mishima, S. M., Anjos, D. S. C., Jorge, J. S. \& Santana, H.P.(2018). Desafios e potencialidades do trabalho de Enfermagem em Consultório na Rua. Revista Latino-Americano de Enfermagem. Ribeirão Preto. (26). 3045.

Costa, S. L., Costa, C. P. V., Gama, I. A., Ping, B. J.K.C.T., Massari, M. G., Paula, T. B. \& Bernardes, A. F. M.(2015). Gestantes em situação de rua no município de Santos, SP: reflexões e desafios para as políticas públicas. Saúde social. São Paulo. 24(3). 1089-1102.

Hallais, J. A. S. \& Barros, N. F. (2015). Consultório na Rua: visibilidades, invisibilidades e hipervisibilidade. Cad. Saúde Pública. Rio de Janeiro. 31(7). 14971504 .

Lima, L. P. M., Santos, A. A. P., Póvoas, F. T. X. \& Silva, F. C. L.(2015).O papel do enfermeiro durante a consulta de pré-natal a gestante usuária de drogas. Espaço da saúde. Londrina. 16 (3). 39-46.

Lira, C. D. G., Justino, J. M. R., Paiva, I. K. S., Miranda, M. G. O. \& Saraiva, A. K. M.(2019). O acesso da população em situação de rua é um direito negado? REME Revista Mineira de Enfermagem. (23). 1157.

Medeiros, C. R. S. \& Cavalcante, P.(2018). A implementação do programa de saúde específico para a população em situação de rua - Consultório na rua: barreiras e facilitadores. Saúde soc. São Paulo. 27(3). 754-768.

Ministério da Mulher, da Família e dos Direitos Humanos. (2020), https://www.gov.br/mdh/pt-br/assuntos/noticias/2019/junho/pessoas-em-situacao-de-rua sao-vitimas-em-889-denuncias-recebidas-pelo-disque-100

Paula, H. C., Daher, D. V., Koopmans, F. F., Faria, M. G. A., Brandão, P. S. \& Scoralick, G. B. F.(2018). A implantação do consultório na rua na perspectiva do cuidado em saúde. Revista Brasileira de Enfermagem. Brasília. 71(supl 6). 2843-2847.

Rizzini, I. \& Couto, R. M. B. (2018). Maternidade adolescente no contexto das ruas. Desidades. Rio de Janeiro. (19). 9-19.

Rosa, A. S. \& Bretas, A. C. P.(2015). A violência na vida de mulheres em situação de rua na cidade de São Paulo. Interface. 19(53). $275-285$.

Santana, C. S., Freitas, I. L. S., Mesquita, K. S. F. de., Araújo, B. R. O. de., Melo, G. B. de.\& Almeida, A. V. A.(2019). Assistência de Enfermagem a Mulher em Situação de Rua no Ciclo Gravídico- Puerperal: Uma Revisão de Literatura. Ciências Biológicas e de Saúde UNIT.Alagoas.5( 2). 71-82.

Silva, F.L. (2019). A cobertura do programa consultório na rua e o impacto no acesso da população em situação de rua aos serviços de saúde. Lume. UFRGS.

Souza, M.T.,Silva, M.D. \& Carvalho, R. (2010). Revisão integrativa: o que é e como fazer. Einstein. 8 (1 Pt 1). $102-6$.

Vale, A. R. \& Vecchia, M. D. (2019). UPA é nós aqui mesmo": as redes de apoio social no cuidado à saúde da população em situação de rua em um município de pequeno porte.Saúde social, São Paulo. 28( 1). 222-234. 